\title{
ANL/MSD/PP- -83083
}

\section{A SIMPLE, CLOSED-FORM EXPRESSION FOR THE X-RAY REFLECTIVITY FROM SUPERLATTICES WITH CUMULATIVE ROUGHNESS*}

David M. Kelly, 1 Eric E. Fullerton, 2 Jacobo Santa-Maria, $1,+\frac{\ddagger}{\ddagger}$ and Ivan K. Schuller ${ }^{1}$

1Physics Department 0319, University of California-San Diego, La Jolla, CA 92093-0319

2 Materials Science Division, Argonne National Laboratory, Argonne, IL 60439

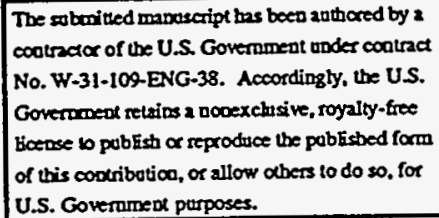

jmc

\section{DISCLAIMER}

\begin{abstract}
This report was prepared as an account of work sponsored by an agency of the United States Government. Neither the United States Government nor any agency thereof, nor any of their employees, makes any warranty, express or implied, or assumes any legal liability or responsibility for the accuracy, completeness, or usefulness of any information, apparatus, product, or process disclosed, or represents that its use would not infringe privately owned rights. Reference herein to any specific commercial product, process, or service by trade name, trademark, manufacturer, or otherwise does not necessarily constitute or imply its endorsement, recommendation, or favoring by the United States Government or any agency thereof. The views and opinions of authors expressed herein do not necessarily state or reflect those of the United States Government or any agency thereof.
\end{abstract}

\footnotetext{
*Work supported by the U.S. Department of Energy , Basic Energy Sciences-Materials Sciences, under contract \#W-31-109-ENG-38.

¥Permanent address: Universidad Complutense, Madrid, Spain.
} 


\section{DISCLAIMER}

Portions of this document may be illegible in electronic image products. Images are produced from the best available original document. 


\title{
A Simple, Closed-Form Expression for the X-ray Reflectivity from Superlattices with Cumulative Roughness
}

David M. Kelly ${ }^{1}$, Eric E. Fullerton ${ }^{2}$, Jacobo Santa-Maria ${ }^{1 \dagger}$, and Ivan K. Schuller ${ }^{1}$

1 University of California, San Diego, Physics Department 0319 La Jolla, Ca 92093-0319

2 Argonne National Laboratory, Material Science Division, Argonne, II 60439

\begin{abstract}
We present a simple closed-form expression for the reflectivity from a multilayer which includes the effects of absorption, refraction, surface and substrate reflections but neglects dynamical effects. This expression reproduces the exact dynamical calculation except for the regions near the critical angle and for intense Bragg reflections. The expression is generalized to include cumulative interface roughness which follows a $t^{1 / 2}$ power-law growth.
\end{abstract}

PACS \#: 61.10.Dp 
$\mathrm{X}$-ray reflectivity is commonly used to study the interface structure and morphology of metallic superlattices. ${ }^{1-7}$ The specular reflectivity is a measure of the average electron density profile of the superlatice and in principle can be used to determine the interdiffusion and average roughness of the interfaces. The most common approach to calculate the low-angle profile is the recursive application of optical theories where the layers are assumed to be continuous media of constant electron density. ${ }^{1-3}$ These theories are equivalent to dynamical calculations and include effects of multiple scattering, absorption, refraction, and surface and substrate reflections. ${ }^{8}$ Although it is straight forward to calculate the reflectivity using this technique, the calculations are time consuming when the superlattice contains a large number of layers. In this paper, we present a simple closed-form expression for the reflectivity of a superlattice which includes the effects of absorption, refraction, and substrate reflections but neglects dynamical effects. The expression is then generalized to include cumulative interface roughness which follows a $t^{1 / 2}$ power-law growth.

The kinematic calculation of the $x$-ray reflectivity from a general $A / B$ superlattice is shown schematically in Fig. 1. The reflectivity of the superlatice is given by summing the reflectivity from each interface ignoring multiple reflections. The surface and substrate reflectivities are given by $r_{S}$ and $r_{\text {sub }}$, and the A-on-B and B-on-A reflectivities are given by $\mathrm{r}_{\mathrm{AB}}$ and $\mathrm{rBA}_{\mathrm{BA}}$ respectively. Each layer is characterized by thickness $t$ and index of refraction $n$ given by: 1

$$
n=1-\frac{\rho_{N} r_{c} \lambda_{X}^{2}}{2 \pi}\left(f_{0}+\Delta f^{\prime}+i \Delta f^{\prime \prime}\right)
$$

where $r_{e}=2.818 \times 10^{-13} \mathrm{~cm}$ is the classical electron radius, $\rho_{n}$ is the atomic density, $f_{0}$ is the atomic scattering power, $\lambda_{X}$ is the $\mathrm{X}$-ray wavelength, and $\Delta f^{\prime}$ and $\Delta f^{\prime \prime}$ are the anomalous dispersion corrections for the atomic scattering factor. Because the index of refraction is slightly less than one, the position of the diffraction peaks will be shifted from the expected Bragg position. The amount of this shift can be directly related to the average 
index of refraction of the superlattice. ${ }^{8}$ The absorption coefficient of the material is proportional to the imaginary component of $n$. The Fresnel reflectivity from a perfect $A-$ on-B interface is proportional to the difference in electron density of the two materials and is given for $s$-polarized $x$-rays by: 1

$$
r=\frac{g_{A}-g_{B}}{g_{A}+g_{B}} \quad \text { where } g=\sqrt{n^{2}-\cos ^{2} \theta}
$$

For $g_{A}<g_{B}$, the reflectivity is negative which implies a $\pi$ phase shift of the $x$-rays upon reflection. The true specular reflectivity will be reduced from the presence of interdiffusion or roughness at the interface. If the average electron density of the interface after lateral averaging can be approximated as an error function with an average width $\sigma$, the reflectivity for the A-on-B and B-on-A interfaces are approximately given by: 4

$$
r_{A B}=\frac{g_{A}-g_{B}}{g_{A}+g_{B}} \exp \left(\frac{q^{2} \sigma_{A B}^{2}}{2}\right) \text { and } r_{B A}=\frac{g_{B}-g_{A}}{g_{B}+g_{A}} \exp \left(\frac{q^{2} \sigma_{B A}^{2}}{2}\right)
$$

where $\mathrm{q}=4 \pi \sin \theta / \lambda_{\mathrm{X}}$ and $\sigma_{\mathrm{AB}}$ and $\sigma_{\mathrm{BA}}$ correspond to the interface width of the A-on-B and B-on-A interfaces respectively. The Fresnel reflectivity is reduced by an effective or 'static' Debye-Waller term when the interface profile is an error function. The damping expression for other types of interface profiles are reported by Stearns. ${ }^{4}$ In this paper, we will assume all interface profiles can be described by an error function.

The kinematic reflectivity of the superlattice shown in Fig. 1 is simple given by:

$$
\begin{aligned}
r= & r_{s}+\left(1-i_{s}\right)^{2}\left[r_{A B} \exp \left(-i \varphi_{B}\right)+r_{B A} \exp \left(-i\left(\varphi_{A}+\varphi_{B}\right)\right)\right. \\
& \left.+r_{A B} \exp \left(-i\left(\varphi_{A}+2 \varphi_{B}\right)\right)+\ldots+r_{s u b} \exp \left(-i\left(N\left(\varphi_{A}+\varphi_{B}\right)\right)\right)\right]
\end{aligned}
$$

where $i_{S}=\left|r_{S}\right|^{2}$ is the intensity lost from the surface reflection. Similar equations have been presented by a number of other authors. 4,5 The $\left(1-\mathrm{i}_{S}\right)^{2}$ term accounts for the loss on intensity from reflections from the surface as the beam enters and leaves the superlattice. This correction is needed near the critical angle when the surface reflectivity is high. We 
have ignored, for simplicity, the loss of intensity resulting from the subsequent interfaces, however this can be easily incorporated within equation (4). $\phi_{A}$ and $\phi_{B}$ determines the resulting phase shifts by passing through layers $A$ and $B$ and is given as $\phi_{A}=4 \pi g_{A} t_{A} \lambda_{X}$ and $\phi_{B}=4 \pi g_{A} t_{A} / \lambda_{X} \cdot 1$ Because $g_{A}$ and $g_{B}$ contain both the real and imaginary components of the index of refraction, the effects of absorption and refraction are included in Eq. (4). With the definitions:

$$
\begin{aligned}
& \alpha=\left(1-i_{s}\right)^{2} \exp \left(-i \varphi_{B}\right)\left(r_{A B}+r_{B A} \exp \left(-i \varphi_{A}\right)\right) \\
& \alpha^{\prime}=\left(1-i_{s}\right)^{2} \exp \left(-i \varphi_{B}\right)\left(r_{A B}+r_{s i b} \exp \left(-i \varphi_{A}\right)\right) \\
& \Phi=\exp \left(-i\left(\varphi_{A}+\varphi_{B}\right)\right)
\end{aligned}
$$

Eq. (4) simplifies to:

$$
r=r_{s}+\alpha+\alpha \Phi+\alpha \Phi^{2}+\ldots+\alpha \Phi^{N-2}+\alpha^{\prime} \Phi^{N-1}
$$

In Eq. (6), $\alpha$ is the reflectivity of a single bilayer and $\Phi$ is the phase shift acquired passing through a bilayer. Since Eq. (6) is a power series in $\Phi$, the final intensity can be written as:

$$
I=|r|^{2}=\left|r_{s}+\alpha \frac{1-\Phi^{N-1}}{1-\Phi}+\alpha^{\prime} \Phi^{N-1}\right|^{2}
$$

Eq. (7) is a simple closed-form expression for the reflectivity which does not require summing over the number of interfaces required for most formulations of this problem. ${ }^{1-9}$ In Fig. (2) we show a direct comparison of Eq. (7) to the standard dynamical formalism for the calculation of a $[\mathrm{Ni}(15 \AA) / \mathrm{W}(20 \AA)]_{10}$ superlattice. Eq. (7) reproduces the exact calculation with only a slight discrepancy near the critical angle. Eq. (7) is easily generalized to unit cells with an arbitrary number of layers.

Eq. (7), in general, does not accurately reproduce the exact calculation when dynamical effects have a significant contribution. This occurs close to the critical angle and when a Bragg reflection has a reflectivity larger than about $10 \%$. In Fig. (2) the first 
Bragg peak has a reflectivity of $12.0 \%$ in the exact calculation and $12.6 \%$ in the present kinematic model. As the reflectivity of the Bragg peak increases, the discrepancies increase, with Eq. (7) overestimating the reflected intensity. The inset in Fig. (2) shows that the discrepancy is larger near the critical angle. If material $B$ has a higher electron density than material A, the critical angle in Eq. (7) will be at a higher angle than the exact calculation and corresponds to the expected position for a single film of material $B$. This is shown in the inset of Fig. (2). If material B has a lower electron density that A, a dip in the reflectivity occurs at the expected critical angle of the lighter material and the reflectivity again drops at the critical angle of the heavier material.

Eq. (7) is derived under the assumption that each bilayer is reproduced exactly. The validity of this assumption depends on the growth process and the constituent materials of the superlattice. In many cases, the roughness which is intrinsic to the growth of a layer will add cumulatively to the roughness of the previous layers.' In this case, the roughness of the interfaces will increase as the superlattice thickness increases. An example of this type of roughness was shown by transmission electron microscopy and $\mathrm{x}$ ray diffraction for sputtered $\mathrm{Nb} / \mathrm{Si}$ superlatices. ${ }^{10}$ In this example, the amount of cumulative roughness depends strongly on the sputtering gas pressure during growth. In many cases, surface roughness of thin films has been found, both theoretically and experimentally, to scale as a power law in the film thickness $t \beta$ where $\beta$ depends on the dynamics of the growth process. $11-13$ In superlattices, this type of roughness has, in general, been modeled as cumulative fluctuations in the layer thickness ${ }^{14-19}$ or increases in the interface width with increased superlattice thickness. ${ }^{19}$ For the low-angle specular reflectivity, these two approaches are equivalent if the scattering amplitude (as opposed to intensity) is averaged over fluctuations in the layer thicknesses. This approach is appropriate for the true specular reflectivity which exhibits long-range lateral coherence. The specular reflectivity is measured experimentally by subtracting the diffuse contribution from the measured spectrum. ${ }^{4}$ In the above formalism it is straight forward to include a 
power law growth of the interface roughness with $\beta=0.5$. Such a model was successfully used to fit the reflectivity of $\mathrm{Nb} / \mathrm{Si}$ multilayers sputtered at various Ar pressures. 19 Within this approximation, the roughness of the A-on-B and B-on-A interfaces in the jth bilayer of the superlattice can be written:

$$
\sigma_{A B j}^{2}=\sigma_{A B}^{2}+j \sigma_{c}^{2} \text { and } \sigma_{B A j}^{2}=\sigma_{B A}^{2}+j \sigma_{c}^{2}
$$

where $\sigma_{\mathrm{c}}$ is the additional cumulative roughness per bilayer. For this definition of the interface roughness Eq. (6) becomes:

$$
\begin{aligned}
r= & r_{s}+\alpha \exp \left(-N q^{2} \sigma_{c}^{2} / 2\right)+\alpha \Phi \exp \left(-(N-1) q^{2} \sigma_{c}^{2} / 2\right)+ \\
& \ldots+\alpha \Phi^{N-2} \exp \left(-2 q^{2} \sigma_{c}^{2} / 2\right)+\alpha^{\prime} \Phi^{N-1} \exp \left(-q^{2} \sigma_{c}^{2} / 2\right)
\end{aligned}
$$

In Eq. (9) we have assumed that the surface roughness has the same $\sigma_{\mathrm{c}}$ contribution as the top bilayer. Eq. (7) is only slightly modified as follows:

$$
I=\left|\exp \left(-N q^{2} \sigma_{c}^{2} / 2\right)\left(r_{s}+\alpha \frac{1-\Phi_{c}^{N-1}}{1-\Phi_{c}}+\alpha^{\prime} \Phi_{c}^{N-1}\right)\right|^{2}
$$

where

$$
\Phi_{c}=\exp \left(-i\left(\varphi_{c}+\varphi_{B}\right)+q^{2} \sigma_{c}^{2} / 2\right)
$$

A $t^{1 / 2}$ scaling of the roughness is expected for cumulative layer thickness fluctuations if they are distributed in a gaussian fashion. ${ }^{19}$ To calculate the intensity for a more general scaling behavior requires calculating the complete summation, Eq. (9), with the appropriate expression for the cumulative roughness contribution with thickness.

Shown in Fig. (3) is the calculated spectra for superlattices with increasing values of $\sigma_{c}$. Fig. (3a) shows the interface roughness per bilayer and Fig. (3b) shows the corresponding reflectivity spectra. As the cumulative roughness increases, the higher order Bragg peaks are reduced in intensity and broadened which is characteristic of this type of roughness. ${ }^{14-19}$ The minima in the calculated spectra result from the W layer which has a much higher electron density than the Ni layers. The minima are periodic with 
a period of $20 \AA$. Similar minima were observed in the experimental spectra from $\mathrm{Pb} / \mathrm{Ge}$ superlatices. 20

In conclusion, we have derived a simple, closed-form, kinematic expression for the reflectivity from a multilayer which includes the effects of absorption, refraction, surface and substrate reflections. This expression reproduces the exact dynamical calculation with only minor discrepancies near the critical angle and for intense Bragg reflections. The expression is then generalized to include cumulative interface roughness which follows a $t^{1 / 2}$ power-law growth.

Work supported by the U.S. Department of Energy, BES-Materials Sciences under contract W-31-109-ENG-38 at Argonne National Laboratry and DE-FG03-87EK-45322 at University of California, San Diego. International travel provided by NATO.

$\grave{i}$ Permanent address: Universidad Complutense, Madrid, Spain

\section{References}

[1] L. G. Parratt, Phys. Rev. 95, 359 (1954).

[2] J. H. Underwood and T. W. Barbee, Appl. Opt. 20, 3027 (1981).

[3] B. Vidal and P. Vincent, Appl. Opt. 23, 1794 (1984).

[4] S. K. Sinha, Physica B 173, 25 (1993).

[5] D. B. Stearns, J. Appl. Phys. 71, 4286 (1992).

[6] D. E. Savage, J. Kleiner, N. Schimke, Y.-H. Phang, T. Jankowski, J. Jacobs, R. Kariotis, and M. B. Lagally, J. Appl. Phys. 69, 1411 (1991).

[7] C. M. Falco, J. M. Slaughter, J. Magn. Magn. Mater. 126, 3 (1993).

[8] M. Born and E. Wolf, Principles of Optics (Pergammon Press, Oxford, 1975), pp. 6770.

[9] P. F. Miceli, D. A. Neumann and H. Zabel, Appl. Phy's. Lett. 48, 24 (1986). 
[10] E. E. Fullerton, I. K. Schuller, and Y. Bruynseraede, MRS Bulletin 17(12), 33 (1992).

[11] M. Kardar, G. Parisi, and Y. Zhang, Phys. Rev. Lett. 56, 889 (1986).

[12] F. Family, Physica A 168, 561 (1990).

[13] H. You, R. P. Chiarello, H. K. Kim, and K. G. Vandervoort, Phys. Rev. Lett. 70, 2900 (1993).

[14] I. K. Schuller, Phys. Rev. Lett 44, 1597 (1980).

[15] W. Sevenhans, M. Gijs, Y. Bruynseraede, H. Homma and I. K. Schuller, Phys. Rev. B 34, 5955 (1986).

[16] J.-P. Locquet, D. Neerinck, L. Stockman, Y. Bruynseraede, and I. K. Schuller, Phys. Rev. B 39, 13338 (1989).

[17] E. E. Fullerton, I. K. Schuller, H. Vanderstraeten, and Y. Bruynseraede, Phys. Rev. B 45, 9292 (1992).

[18] A. P. Payne and B. M. Clemens, Phys. Rev. B 47, 2289 (1993).

[19] E. E. Fullerton, J. Pearson, C. H. Sowers, S. D. Bader, X. Z. Wu, and S. K. Sinha, Phys. Rev. B 48, 17432 (1993).

[20] D. Neerinck, H. Vanderstraeten, L. Stockman, J. -P. Locquet, Y. Bruynseraede, and I. K. Schuller, J. Phys. Condens. Matter 2, 4111 (1990). 


\section{Figure Captions}

Figure 1: Schematic representation of kinematic diffraction from a superlattice.

Figure 2: Model calculations of a $[\mathrm{Ni}(15 \AA) / \mathrm{W}(20 \AA)]_{10}$ superlattice where the interface roughness parameters $\sigma_{S}=\sigma_{A B}=\sigma_{B A}=\sigma_{s u b}=2 \AA$. The circles are the full dynamical calculation described in Ref. 3 and the solid line is the kinematic approximation given by Eq. (7). Shown in the inset is an expanded view near the critical angle.

Figure 3: Model calculations for the same $[\mathrm{Ni}(15 \AA) / \mathrm{W}(20 \AA)]_{10}$ superlattice described in Fig. 2 with additional cumulative roughness as described by Eq. (8). (a)Interface roughness $\sigma$ as a function of the number of bilayers for $\sigma_{C}=0.0,0.5,1.0$ and $2.0 \AA$. (b) Calculated low-angle spectra for interface roughness distributions shown in (a). The spectra are offset by two decades for clarity and the top spectra corresponds to $\sigma_{c}=0.0 \AA$ and bottom spectra to $\sigma_{c}=2.0 \AA$. 


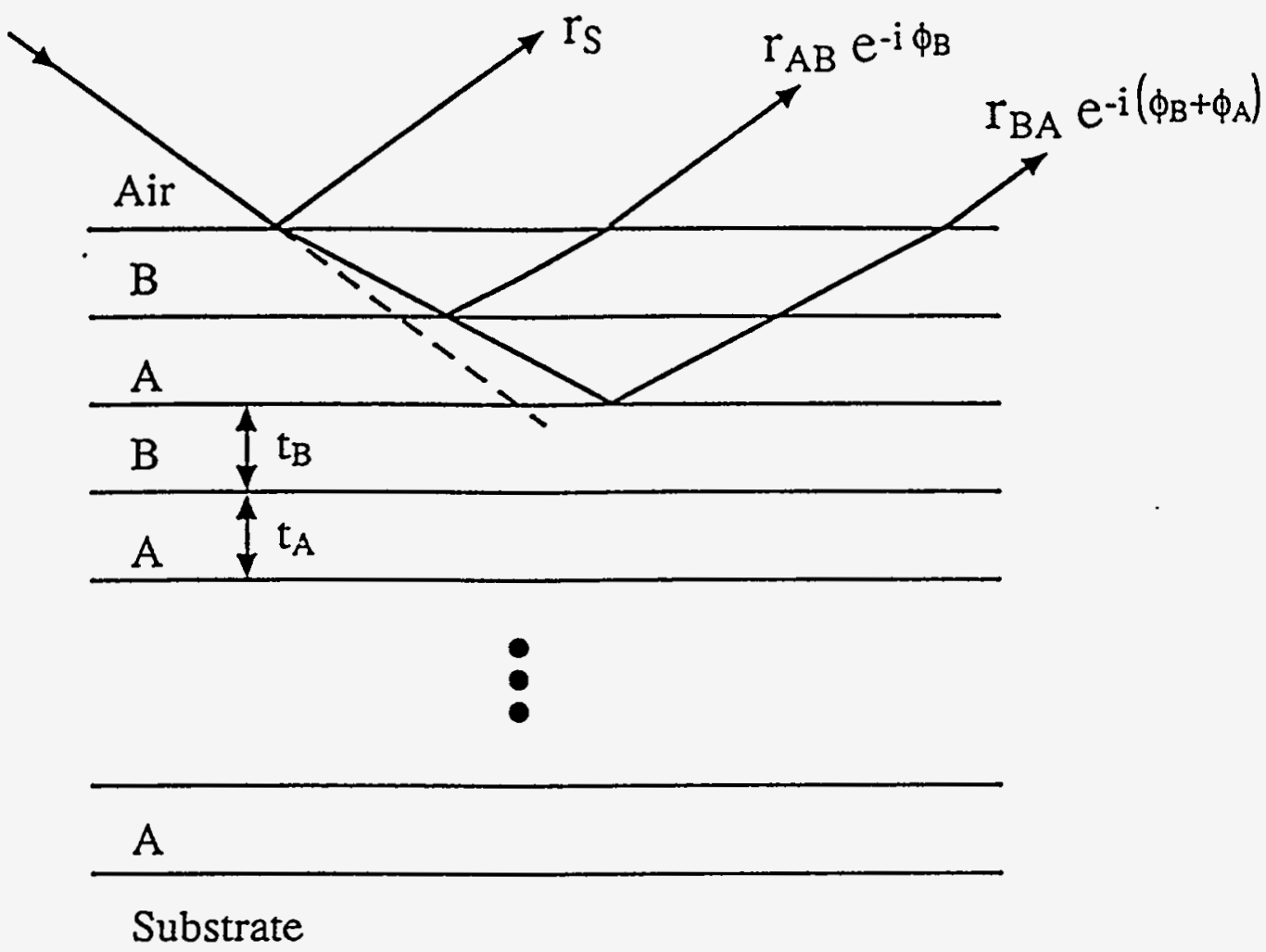




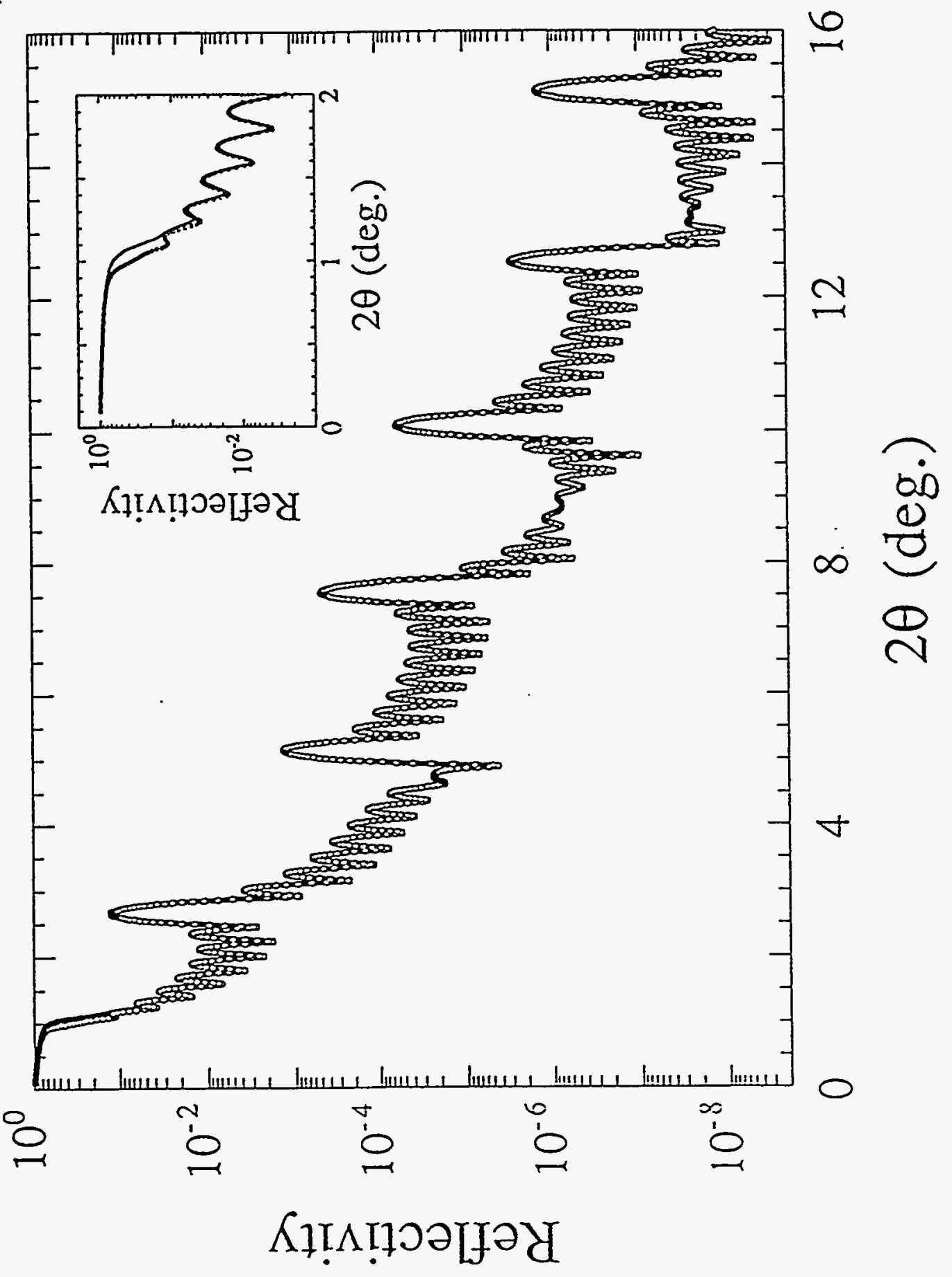



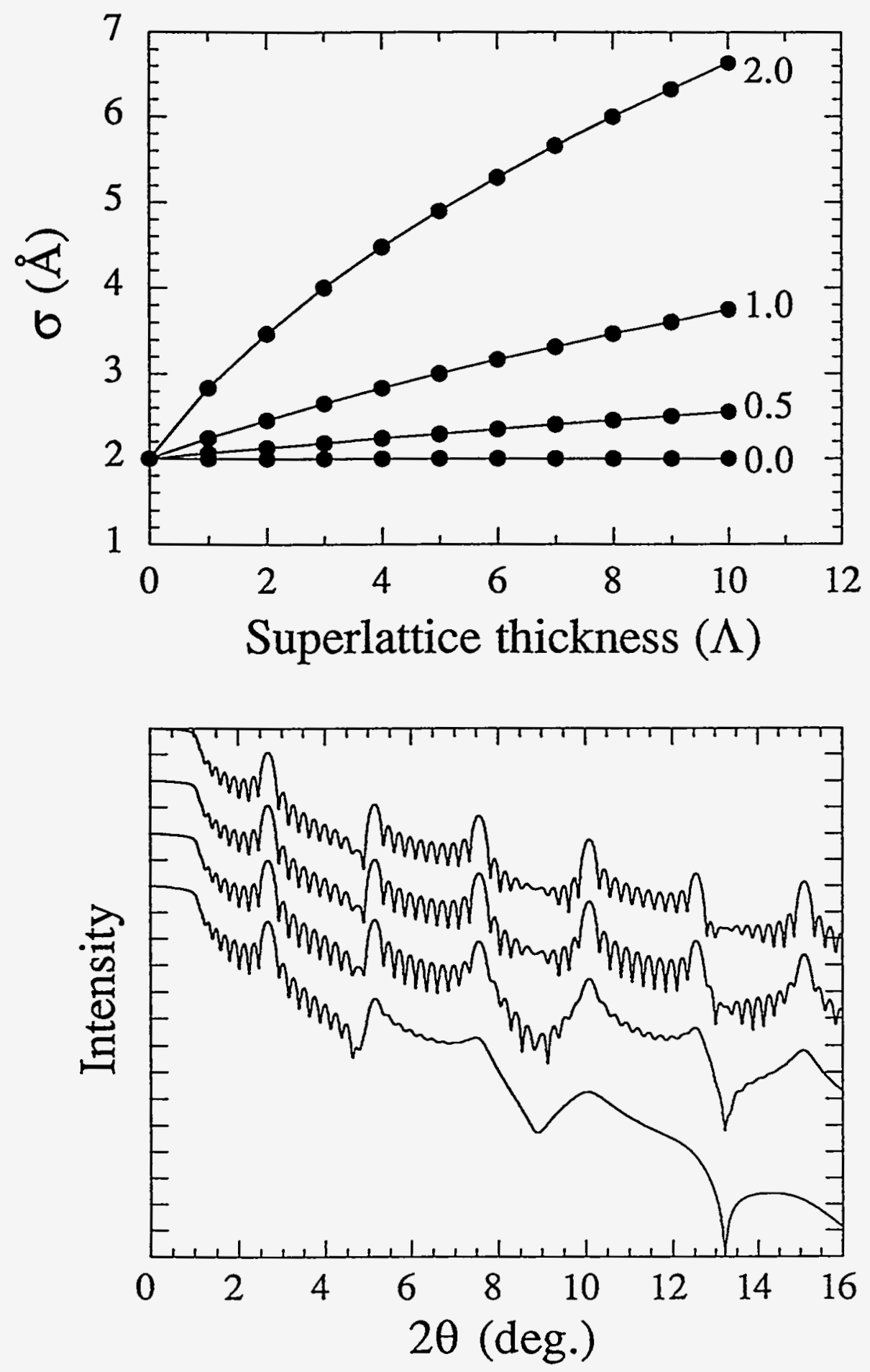